\title{
P04-4-20 Poster session
}

\section{Treating Acute Myeloid Leukaemia: Overcoming the Differentiation Block}

\section{Purnima Kumar}

Department of Pharmacology, University of Oxford, UK

Acute myeloid Leukemia (AML) is a well characterized hematological malignancy that is marked by uncontrolled proliferation, decreased apoptosis and a block in differentiation leading to an increase of immature cells.

AML comprises of a vast genetic variability and the French American British Classification (FAB) divides it from M0 to M7 based on the type of cell from which it develops and the maturity of the cells. Even though the genetics of the subtypes have been widely studied, the therapy hasn't changed in the last years. Current AML treatment consists of intensive chemotherapy and hematopoietic stem cell transplantation (HSCT). Along with these treatments being extremely cytotoxic for elderly patients, refractory AML and early relapse are issues which still need to be addressed.

Since the different genotypes of AML come with a common block in differentiation thus overcoming the differentiation blockade has emerged as an attractive therapeutic strategy.

In a small phenotypic screen, compounds were identified which increased the expression of CD11b - a cell surface marker known to indicate differentiation to myeloid lineages. In an assay with six genetically distinct AML cell lines, clinical histone deacetylase inhibitors (HDACi) and some novel analogues were observed to cause an upregulation in the CD11b expression. These were further validated by a change in cell morphology and a block in

proliferation. The novel analogues also caused a cell cycle arrest at G1 phase. The novel compounds were successfully resynthesized along with analogues devoid of HDAC inhibiting activity as mechanistic probes. Further work through western blots is being done to confirm if the effects observed above were due to the acetylation of histones or another mechanism independent of HDAC inhibition.

Very little has been studied about the genotypic effects seen in AML with HDACi. These novel compounds along with their potential inactive analogues could help to monitor the target engagement in the cells and identify the pharmacological effect on perturbation of the HDACs or any other target. 